\title{
Editorial: Microbiology of Ethnic Fermented Foods and Alcoholic Beverages of the World
}

\author{
Jyoti P. Tamang ${ }^{1 *}$, Wilhelm H. Holzapfel ${ }^{2}$, Dong H. Shin ${ }^{3}$ and Giovanna E. Felis ${ }^{4}$ \\ ${ }^{1}$ Microbiology, Sikkim University, Gangtok, India, ${ }^{2}$ Microbiology, Handong Global University, Pohang, South Korea, \\ ${ }^{3}$ Microbiology, Chonbuk National University, Jeonju, South Korea, ${ }^{4}$ Microbiology, University of Verona, Verona, Italy
}

Keywords: editorial, fermented foods, food microbiology, next generation sequence, global

Editorial on the Research Topic

Microbiology of Ethnic Fermented Foods and Alcoholic Beverages of the World

Approximately there may be around 5,000 varieties of common and uncommon fermented foods and alcoholic beverages in the world. Global fermented foods are classified into 9 major groups on the basis of substrates (raw materials) used from plant/animal sources: fermented cereals, fermented vegetables and bamboo shoots, fermented legumes, fermented roots/tubers, fermented milk products, fermented and preserved meat products, fermented, dried, and smoked fish products, miscellaneous fermented products, and alcoholic beverages. Fermented foods are the hubs of consortia of microorganisms, which transform the chemical constituents of raw materials of plant/animal sources during in situ/ex situ fermentation, thereby enhance the nutritional value with health-promoting bioactive compounds to consumers.

Edited by:

Aldo Corsetti,

University of Teramo, Italy

Reviewed by:

Rosalba Lanciotti,

Università di Bologna, Italy

*Correspondence:

Jyoti P. Tamang

jyoti_tamang@hotmail.com

Specialty section:

This article was submitted to

Food Microbiology,

a section of the journal

Frontiers in Microbiology

Received: 29 April 2017

Accepted: 06 July 2017

Published: 20 July 2017

Citation:

Tamang JP, Holzapfel WH, Shin DH and Felis GE (2017) Editorial: Microbiology of Ethnic Fermented

Foods and Alcoholic Beverages of the World. Front. Microbiol. 8:1377. doi: 10.3389/fmicb.2017.01377
Common genera of the lactic acid bacteria isolated from various fermented foods globally are Alkalibacterium, Carnobacterium, Enterococcus, Lactobacillus, Lactococcus, Leuconostoc, Oenococcus, Pediococcus, Streptococcus, Tetragenococcus, Vagococcus and Weissella. Species of Bacillus are reported for alkaline-fermented foods of Asia and Africa. The association of several species of Kocuria, Micrococcus (members of the Actinobacteria), and Staphylococcus (belonging to the Firmicutes) has been reported for fermented milk, fermented meat, and fish products. Species of Bifidobacterium, Brachybacterium, Brevibacterium, and Propionibacterium have been isolated from cheese and species of Arthrobacter and Hafnia from meat fermentation. Enterobacter cloacae, Klebsiella pneumoniae, K. pneumoniae subsp. ozaenae, Haloanaerobium, Halobacterium, Halococcus, Propionibacterium, and Pseudomonas, are also present in numerous fermented foods.

Genera of yeasts reported for fermented foods, alcoholic beverages, and non-food mixed amylolytic starters are Brettanomyces, Candida, Cryptococcus, Debaryomyces, Dekkera, Galactomyces, Geotrichum, Hansenula, Hanseniaspora, Hyphopichia, Issatchenkia, Kazachstania, Kluyveromyces, Metschnikowia, Pichia, Rhodotorula, Rhodosporidium, Saccharomyces, Saccharomycodes, Saccharomycopsis, Schizosaccharomyces, Sporobolomyces, Torulaspora, Torulopsis, Trichosporon, Yarrowia, and Zygosaccharomyces. Major roles of filamentous fungi in fermented foods and alcoholic beverages are mainly production of enzymes and also degradation of anti-nutritive factors. Species of Actinomucor, Amylomyces, Aspergillus, Monascus, Mucor, Neurospora, Parcilomyces, Penicillium, Rhizopus, and Ustilago are reported for many fermented foods, Asian non-food amylolytic starters and alcoholic beverages.

Direct DNA extraction from samples of fermented foods, commonly called culture-independent methods, is nowadays frequently used in food microbiology to profile both cultivable and 
uncultivable microbial populations from fermented foods. Amplified ribosomal DNA restriction analysis (ARDRA) and denaturing gradient gel electrophoresis (DGGE) techniques developed to profile microbial communities directly from fermented foods, and are based on sequence-specific distinctions of $16 \mathrm{~S}$ rDNA or $26 \mathrm{~S}$ rDNA amplicons produced by PCR.

Application of next generation sequencing (NGS) such as metagenomic approaches by using parallel pyrosequencing of tagged 16S rRNA gene amplicons provide information on microbial communities as profiled in kimchi, a naturally fermented vegetable product of Korea, nukadoko, a fermented rice bran of Japan, narezushi, a fermented salted fish and cooked rice of Japan, and ben-saalga, a traditional gruel of pearl millet of Burkina Faso. A proteomics identification method based on protein profiling using matrix-assisted laser desorption ionizingtime of flight mass spectrometry (MALDI-TOF MS) is used to identify bacteria in fermented foods. NGS has revealed the new dimension of microbial ecology comprising both cultivable and uncultivable microorganisms in many ethnic fermented foods and beverages of the world.
This e-book is a compilation of 15 originals and reviews papers written by 94 authors. We tried to represent the main Asian, African, European, and American fermented foods and alcoholic beverages.

\section{AUTHOR CONTRIBUTIONS}

JT prepared a draft concept on Resource Topic of the present ebook and list of authors for papers, supplemented and corrected by WH, DS, and GF. JT was the main corresponding Editor, however, WH, DS, and GF also helped to provide the names of referrers, etc.

Conflict of Interest Statement: The authors declare that the research was conducted in the absence of any commercial or financial relationships that could be construed as a potential conflict of interest.

Copyright () 2017 Tamang, Holzapfel, Shin and Felis. This is an open-access article distributed under the terms of the Creative Commons Attribution License (CC BY). The use, distribution or reproduction in other forums is permitted, provided the original author(s) or licensor are credited and that the original publication in this journal is cited, in accordance with accepted academic practice. No use, distribution or reproduction is permitted which does not comply with these terms. 